\title{
Efeito do Trinexapac-EThyl na Anatomia Foliar de Quatro ESPÉCIES DE GRAMA ${ }^{1}$
}

\author{
Trinexapac-Ethyl Effect on the Leaf Anatomy of Four Turfgrass Species
}

COSTA, N.V. ${ }^{2}$, MARTINS, D. ${ }^{3}$, RODELLA, R.A. ${ }^{4}$, RODRIGUES, A.C.P. ${ }^{5}$ e CARDOSO, L.A. ${ }^{5}$

\begin{abstract}
RESUMO - Os reguladores de crescimento podem retardar o desenvolvimento vegetativo das plantas de gramas e, assim, reduzir a frequência de cortes; contudo, existem poucas informações referentes aos efeitos desses produtos sobre as estruturas da anatomia foliar. Dessa forma, o presente trabalho teve por objetivo avaliar os efeitos da aplicação sequencial de duas doses de trinexapac-ethyl sobre a anatomia foliar das espécies de gramas São Carlos (Axonopus compressus), Batatais (Paspalum notatum), Santo Agostinho (Stenotaphrum secundatum) e Esmeralda (Zoysiajaponica). Os tratamentos utilizados foram constituídos de duas aplicações sequenciais de trinexapac-ethyl nas doses de 56,5+56,5 e 113,0+113,0 g ha-1, além de uma testemunha sem aplicação, para cada espécie avaliada. Os gramados foram cortados à altura de $3 \mathrm{~cm}$, com auxilio de um aparador de grama motorizado, e, em seguida, foram realizadas as aplicações dos tratamentos. Após 20 dias da primeira aplicação de trinexapac-ethyl, as parcelas foram novamente aparadas à altura de $3 \mathrm{~cm}$ e foi realizada a segunda aplicação dos tratamentos. O delineamento experimental utilizado foi o de blocos ao acaso, com quatro repetições. Aos 70 dias após a segunda aplicação dos tratamentos, foram realizadas as amostragens do material foliar, para as quatro espécies estudadas. Os dados das variáveis quantitativas foram submetidos ao teste estatístico multivariado de análise de componentes principais. Os resultados evidenciaram a formação de três e dois grupos principais, para os caracteres da região da quilha (nervura mediana) e da região da asa (situada entre a nervura mediana e a margem do limbo foliar), respectivamente. De modo geral, em cada formação dos agrupamentos, os tratamentos com trinexapac-ethyl apresentaram maior similaridade entre si, em relação às respectivas testemunhas. Conclui-se que a aplicação sequencial de trinexapac-ethyl alterou algumas estruturas anatômicas da região da quilha e da asa do limbo foliar das espécies de gramas estudadas.
\end{abstract}

Palavras-chave: anatomia quantitativa, gramado, regulador vegetal, análise multivariada.

\begin{abstract}
Plant growth regulators can retard the growth of turfgrasses, reducing the frequency of cuts; however, there is little information on the effects of such products on the structures of the leaf anatomy. Thus, this study aimed to evaluate the effect of sequential application of two rates of trinexapac-ethyl on the leaf anatomy of the turfgrass species Broadleaf Carpetgrass (Axonopus compressus), Bahiagrass (Paspalum notatum), St. Augustinegrass (Stenotaphrum secundatum) and Korean Lawngrass (Zoysia japonica). The treatments were two sequential applications of trinexapac-ethyl at two rates, $\left(56.5+56.5\right.$ and113. $\left.+113.0 \mathrm{~g} \mathrm{ha}^{-1}\right)$, plus a control without spraying, for each species evaluated. The turfgrasses were cut with a motorized grass cutter up to $3 \mathrm{~cm}$ height, and sprayed 20 days after the treatments. After the first trinexapac-ethyl application, the plots were cut again and the second application of the treatments was performed. The experiments were arranged in a completely randomized block design with four replications. Seventy days after the second application of the treatments, samples were collected from the leaf material of the four species studied. The data of the quantitative variables were submitted to the
\end{abstract}

Recebido para publicação em 1.6.2009 e na forma revisada em 3.9.2010.

2 Professor Adjunto da UNIOESTE/CCA, 85960-000 Marechal Cândido Rondon-PR, <neumarciovc@hotmail.com>; ${ }^{3}$ Professor Adjunto, Faculdade de Ciências Agrárias, Universidade Estadual de São Paulo - FCA/UNESP, Fazenda Lageado, Caixa Postal 237, 18603-970 Botucatu-SP; ${ }^{4}$ Professor Adjunto do IBB/UNESP, Botucatu-SP; ${ }^{5}$ Doutorando em Agronomia do Dep. de Produção Vegetal, FCA/UNESP, Botucatu-SP.

Planta Daninha, Viçosa-MG, v. 28, n. 3, p. 551-560, 2010 
multivariate method of principal components analysis. The results showed the formation of three and two main groups for the characters of the keel region (midrib) and wing region (located between the midrib and the leaf margin), respectively. In general, in each group, the trinexapac-ethyl treatments showed greater similarity, compared with the control. Thus, it was concluded that the sequential application of trinexapac-ethyl changed some leaf anatomical structures of the keel and wing regions in the turfgrass species studied.

Keywords: quantitative anatomy, turfgrasses, growth regulator, multivariate analysis.

\section{INTRODUÇÃO}

Os reguladores vegetais utilizados em gramas podem reduzir a emissão de inflorescências e a altura das plantas sem prejudicar a densidade ou causar dano visivel ao gramado, como pontos necróticos, descoloração ou afinamento, mantendo-se uma alta qualidade da área tratada. Eles também diminuem o número de operações de corte (roçadas) realizadas durante o período de crescimento (primavera-verão), reduzindo os custos de manutenção do gramado (Johnson, 1992; Jiang \& Fry, 1998; Lickfeldt et al., 2001).

O trinexapac-ethyl é um regulador vegetal do tipo II, comumente utilizado nos Estados Unidos para reduzir o crescimento vegetativo de gramados, pertencente ao grupo químico das cicloexanodionas e apresenta estrutura similar à dos herbicidas graminicidas sethoxydim e clethodim (Johnson, 1994; Fagerness \& Penner, 1998c; Heckman et al., 2001). Os reguladores do tipo II atuam como inibidores da biossintese de giberelinas, interrompendo o alongamento celular (Ervin \& Koski, 2001).

Segundo Adams et al. (1992), o mecanismo de ação do trinexapac-ethyl está relacionado com a inativação da enzima $\mathrm{GA}_{20} 3 \beta$-hydroxilase, devido provavelmente à competição entre o regulador vegetal e o 2-oxogluterato pelo cossubstrato $\mathrm{Fe}^{+2} /$ ascorbato-dependente dioxygenase, reduzindo o nivel de giberelinas ativas, principalmente $\mathrm{GA}_{1}$.

O trinexapac-ethyl pode promover a inibição do crescimento por um periodo de quatro a seis semanas após o tratamento, em várias espécies de grama (Johnson, 1992, 1993, 1994, 1997; Fagerness \& Penner, 1998a,b; Freitas et al., 2002). Costa et al. (2009) verificaram que a aplicação sequencial de trinexapac-ethyl nas gramas São Carlos (Axonopus compressus),
Batatais (Paspalum notatum), Santo Agostinho (Stenotaphrum secundatum) e Esmeralda (Zoysia japonica) pode reduzir a necessidade de cortes por um período de até 55 dias.

Os trabalhos realizados por Green et al. (1990), Jiang \& Fry (1998) e Lickfeldt et al. (2001) destacam que a utilização dos reguladores vegetais pode diminuir a demanda de água pela planta e auxiliar no desempenho dos fungicidas aplicados nos gramados.

O trinexapac-ethyl pode ser usado para melhorar a densidade, a coloração e o desempenho de gramados utilizados em áreas esportivas (campos de futebol, golfe, beisebol, polo, tênis, etc.), áreas residenciais (jardins) e áreas públicas (parques, praças, prédios públicos, etc.). Além disso, esse produto também pode ser utilizado em áreas onde as operações de roçada oferecem riscos de acidentes, como locais com topografia acentuada e margens de rodovias com tráfego intenso de veículos (Dernoeden, 1984; Bush et al., 1998).

A anatomia das plantas, principalmente das folhas, pode influenciar na quantidade interceptada e retida dos produtos pulverizados, bem como nos processos de absorção (McDaniel et al., 1990; Procópio et al., 2003; Monquero et al., 2005). Dessa forma, o estudo da estrutura foliar das espécies de gramas pode ser útil na identificação de caracteres anatômicos que podem influenciar na absorção e, em consequência disso, no efeito dos reguladores de crescimento vegetal nas plantas. Além disso, a descrição anatômica pode servir como uma ferramenta adicional na identificação de espécies ou até mesmo de variedades.

De acordo com Ervin \& Koski (2001) e Tan \& Qian (2003), os efeitos do trinexapac-ethyl na composição anatômica e bioquímica da folha de espécies de grama, assim como suas 
implicações após o tratamento, são poucos estudados.

O presente trabalho teve por objetivo avaliar os efeitos da aplicação sequencial de duas doses de trinexapac-ethyl sobre a anatomia foliar das principais espécies de gramas cultivadas no Brasil.

\section{MATERIAL E MÉTODOS}

Foram avaliadas as espécies de gramas São Carlos (Axonopus compressus), Batatais (Paspalum notatum), Santo Agostinho (Stenotaphrum secundatum) e Esmeralda (Zoysia japonica). Para cada espécie de grama foi utilizado o delineamento experimental de blocos ao acaso, com quatro repetições. As parcelas foram constituídas de $2 \mathrm{~m}$ de largura por $2 \mathrm{~m}$ de comprimento.

Os gramados foram cortados à altura de $3 \mathrm{~cm}$, com auxílio de um aparador de grama motorizado; em seguida, realizaram-se as aplicações dos tratamentos.

Os tratamentos foram aplicados após 26 meses do plantio das mudas (3/2/2006), sendo realizadas duas aplicações sequenciais de trinexapac-ethyl (Primo Maxx ${ }^{\mathrm{TM}}, 113,0 \mathrm{~g} \mathrm{~L}^{-1}$ - produto comercial) nas doses de 56,5+56,5 e $113,0+113,0 \mathrm{~g} \mathrm{ha}^{-1}$, além de uma testemunha sem aplicação para cada espécie avaliada. As doses de trinexapac-ethyl foram determinadas com base nos resultados de estudos preliminares.

As aplicações sequenciais foram realizadas com intervalo de 20 dias, no período da manhã, em condições de temperatura de $24{ }^{\circ} \mathrm{C}$, solo úmido e umidade relativa do ar em torno de $80 \%$. Na aplicação do regulador vegetal foi utilizado um pulverizador costal, pressurizado a $\mathrm{CO}_{2}$ e munido de barra com quatro pontas de jato plano XR 110.02VS, espaçadas entre si de $50 \mathrm{~cm}$, com consumo de $200 \mathrm{~L} \mathrm{ha}^{-1}$.

Após a primeira aplicação do trinexapacethyl, as parcelas foram novamente aparadas à altura de $3 \mathrm{~cm}$ e realizou-se a segunda aplicação dos tratamentos. Durante as aplicações, as parcelas foram protegidas lateralmente com biombos de lona.

Para se proceder à análise anatômica da folha das quatro espécies de gramas estudadas, as amostras foliares foram coletadas aos 70 dias após a segunda aplicação dos tratamentos. As amostras de folhas inteiramente expandidas foram coletadas ao acaso no centro das parcelas, obtendo-se quatro repetições.

As amostras foliares do terço médio do limbo foliar, compreendendo, de acordo com a terminologia descrita para gramíneas por Metcalfe (1960), as regiões da quilha (nervura mediana) e da asa (região situada entre a nervura mediana e a margem do limbo foliar), foram fixadas em $\mathrm{FAA}_{50}$, durante 48 horas, sendo, posteriormente, estocadas em álcool 70\% (Johansen, 1940).

As amostras foliares foram desidratadas, em série etílica crescente, e em seguida incluídas em resina histológica (Historesin Leica), de acordo com metodologia proposta por Gerrits (1991). Cortes transversais, de $8 \mu \mathrm{m}$ de espessura, foram realizados em micrótomo rotativo (Leica RM2145), os quais foram corados com Azul de Toluidina 0,05\% (O’Brien et al., 1964) e montados em resina sintética (Permount).

Para a quantificação das áreas das estruturas anatômicas das regiões da quilha e da asa do limbo foliar, das quatro repetições avaliadas, foram desenhados os limites e contornos dos tecidos de cada amostra foliar coletada, com o auxílio de microscópio de projeção (Zeiss - Jena); as mensurações das áreas foram feitas com auxílio de mesa digitalizadora acoplada a computador equipado com o programa de Sistema de Planimetria (SPLAN). Os valores obtidos da área das estruturas foliares, representando a média das quatro repetições avaliadas, foram expressos em porcentagems (\%), considerando-se como $100 \%$ a área total de cada região (quilha e asa) analisada, conforme a metodologia proposta por Souza et al. (2005). Os seguintes caracteres quantitativos das regiões da quilha e da asa foram avaliados: porcentagens da epiderme das faces adaxial e abaxial, feixe vascular, bainha do feixe vascular, esclerênquima, parênquima e espessura foliar. Foi também determinado o número de feixes vasculares em $360 \mu \mathrm{m}$ linear da estrutura foliar da região da asa.

Os dados obtidos dos sete caracteres estruturais quantitativos da região da quilha 
e dos oito caracteres estruturais quantitativos da região da asa do limbo foliar foram submetidos à análise multivariada, compreendendo a análise de componentes principais baseada na matriz de covariâncias (Sneath \& Sokal, 1973).

A análise de componentes principais foi empregada para verificar a capacidade discriminatória dos caracteres originais no processo de formação dos agrupamentos, sendo o resultado dessa análise apresentado na forma de dispersão gráfica. Os componentes principais (Y) a serem selecionados devem satisfazer no mínimo $70 \%$ da informação acumulada. Para o processamento das análises estatísticas, foi utilizado o programa NTSYS (Numerical Taxonomical System), versão 1.0.

\section{RESULTADOS E DISCUSSÃO}

Na Tabela 1, podem-se observar os coeficientes de correlação entre os sete caracteres estruturais quantitativos da região da quilha do limbo foliar e os dois primeiros componentes principais $\left(\mathrm{Y}_{1}\right.$ e $\left.\mathrm{Y}_{2}\right)$. No geral, esses componentes foram responsáveis por $86,63 \%$ da informação acumulada pelos caracteres avaliados, evidenciando que são indicadores eficientes de dissimilaridade, ou seja, apresentaram-se como caracteres diferenciadores entre as espécies de gramas, mesmo após a aplicação do regulador de crescimento trinexapac-ethyl.

A intensidade da contribuição desses caracteres quantitativos, para a discriminação das espécies e das doses de trinexapac-ethyl, está relacionada à ocorrência de maiores valores absolutos em $\mathrm{Y}_{1}$ e $\mathrm{Y}_{2}$, os quais se caracterizam por apresentar maior poder discriminatório. Dessa forma, pode-se verificar para o primeiro componente principal $\left(\mathrm{Y}_{1}\right)$ que os caracteres que mais contribuíram para a discriminação dos tratamentos de trinexapacethyl entre as espécies de gramas foram: porcentagens de bainha do feixe vascular, de feixe vascular, de parênquima e a espessura foliar. O componente $Y_{1}$ apresentou 62,98\% de informação retida, a qual é considerada satisfatória para explicar a contribuição dos caracteres analisados na formação dos agrupamentos dos tratamentos aplicados sobre as quatro espécies de gramas avaliadas. Os caracteres relacionados com as porcentagens das faces adaxial e abaxial da epiderme e de esclerênquima evidenciaram baixo poder discriminatório.

Para o segundo componente principal $\left(\mathrm{Y}_{2}\right)$, pode-se constatar que a informação retida foi de 23,65\%, cujo valor é considerado baixo, bem como nenhum dos caracteres anatômicos avaliados contribuiu para discriminar os tratamentos de trinexapac-ethyl aplicados nas quatro espécies de grama avaliadas.

A dispersão gráfica referente à análise dos componentes principais (Figura 1) dos sete caracteres estruturais da região da quilha do limbo foliar evidenciou que os caracteres com maior poder discriminatório foram os responsáveis pelos agrupamentos formados entre as espécies com os respectivos tratamentos de trinexapac-ethyl, constituindo, dessa forma, três grupos principais.

O primeiro grupo foi formado pelos tratamentos aplicados nas espécies $S$. secundatum e $P$. notatum, sendo ele constituído de dois subgrupos, compostos pelos tratamentos de trinexapac-ethyl com as respectivas testemunhas (subgrupo $1=$ tratamentos aplicados em $S$. secundatum; e subgrupo 2 = tratamentos aplicados em $P$. notatum). Da mesma forma, o segundo e o terceiro grupo foram constituídos pelos tratamentos aplicados nas espécies A. compressus e $Z$. japonica, respectivamente. Assim, as espécies do grupo 1 e as dos grupos 2 e 3 apresentaram alto grau de dissimilaridade entre si, ao contrário das espécies do grupo 1 ( $S$. secundatum e $P$. notatum), que

Tabela 1 - Coeficientes de correlação entre os sete caracteres anatômicos quantitativos da região da quilha do limbo foliar de quatro espécies de grama e os dois primeiros componentes principais

\begin{tabular}{|c|l|c|c|}
\hline $\mathrm{N}^{\mathrm{o}}$ & \multicolumn{1}{|c|}{ Caracteres } & $\mathrm{Y}_{1}$ & $\mathrm{Y}_{2}$ \\
\hline 1 & \% Epiderme adaxial & 0,3245 & 0,5737 \\
\hline 2 & \% Epiderme abaxial & $-0,1883$ & $-0,4709$ \\
\hline 3 & \% Bainha do feixe vascular & 0,9886 & $-0,0245$ \\
\hline 4 & \% Feixe vascular & 0,8960 & $-0,3012$ \\
\hline 5 & \% Esclerênquima & $-0,5293$ & $-0,5001$ \\
\hline 6 & \% Parênquima & $-0,9945$ & $-0,0256$ \\
\hline 7 & Espessura foliar $(\mathrm{m})$ & $-0,7028$ & 0,3271 \\
\hline & \% Informação retida & 62,98 & 23,65 \\
\hline & \% Informação acumulada & 62,98 & 86,63 \\
\hline
\end{tabular}




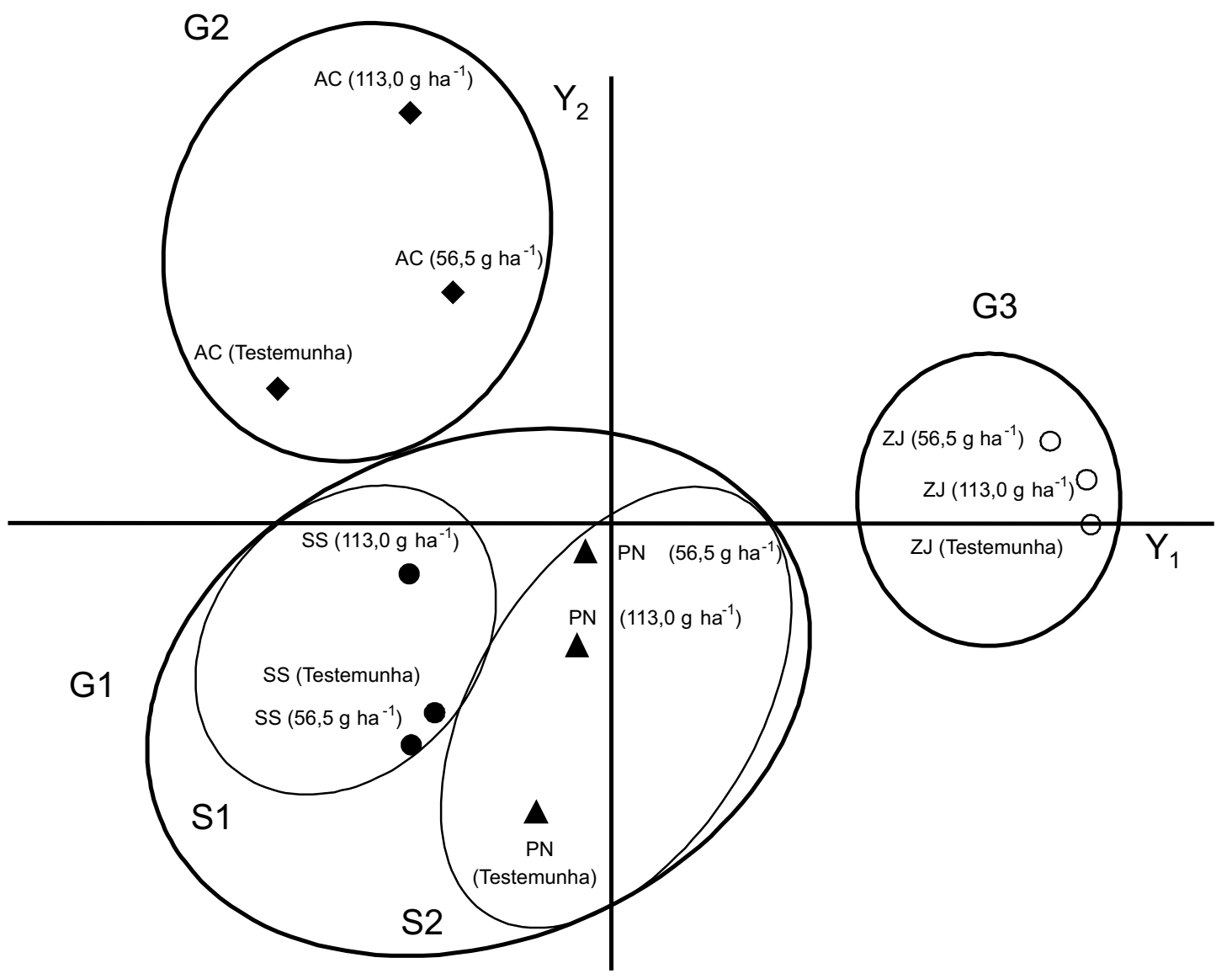

Figura 1 - Dispersão gráfica das quatro espécies de grama e dos tratamentos com trinexapac-ethyl, utilizando-se os dois primeiros componentes principais $\left(\mathrm{Y}_{1}\right.$ e $\left.\mathrm{Y}_{2}\right)$, para o conjunto dos sete caracteres anatômicos quantitativos da região da quilha do limbo foliar. $\mathrm{AC}=$ Axonopus compressus $; \mathrm{SS}=$ Stenotaphrum secundatum, $\mathrm{PN}=$ Paspalum notatum, , ZJ = Zoysia japonica $. \mathrm{G} 1=$ grupo 1 ; $\mathrm{G} 2=$ grupo $2 ; \mathrm{G} 3=$ grupo $3 ; \mathrm{S} 1=$ subgrupo $1 ; \mathrm{e} \mathrm{S} 2=$ subgrupo 2.

mostraram características similares, com base nos caracteres anatômicos quantitativos avaliados.

Observou-se ainda, em cada grupo e subgrupo, que ambas as doses de trinexapac-ethyl apresentaram características mais similares entre si que em relação às testemunhas, com exceção dos tratamentos pertencentes ao subgrupo 1. Esse resultado indica que a aplicação sequencial de trinexapac-ethyl nas espécies de gramas pode alterar alguns caracteres anatômicos da região da nervura mediana do limbo foliar.

Observando os coeficientes de correlação de $\mathrm{Y}_{1}$ na Tabela 1, associados à dispersão gráfica (Figura 1), pode-se constatar que as espécies $S$. secundatum e $P$. notatum, constituintes do grupo 1 , e a espécie $A$. compressus, constituinte do grupo 2, apresentaram os menores valores dos caracteres relativos à bainha do feixe vascular e ao feixe vascular e os maiores valores de parênquima e espessura foliar. Entretanto, o grupo 3 (Z. japonica) apresentou os maiores valores dos caracteres relativos à bainha do feixe vascular e ao feixe vascular, bem como os menores valores dos caracteres referentes ao parênquima e à espessura foliar.

No que se refere aos coeficientes de correlação do $\mathrm{Y}_{2}$ (Tabela 1) e à dispersão gráfica dos tratamentos de trinexapac-ethyl em cada espécie estudada (Figura 1), verificou-se que as espécies $S$. secundatum e $P$. notatum, pertencentes ao grupo 1, apresentaram menores valores referentes à porcentagem de epiderme adaxial; esse fato pode explicar a alta 
similaridade entre as espécies deste grupo, bem como a alta dissimilaridade que ocorreu entre os demais grupos obtidos.

Na Tabela 2 estão demonstrados os coeficientes de correlação entre os oito caracteres anatômicos quantitativos da região da asa $\mathrm{e}$ os dois primeiros componentes principais $\left(\mathrm{Y}_{1}\right.$ e $\mathrm{Y}_{2}$ ). No geral, esses componentes foram responsáveis por $83,80 \%$ da informação acumulada pelos caracteres avaliados, evidenciando que eles são indicadores eficientes de dissimilaridade entre os tratamentos do regulador de crescimento trinexapac-ethyl aplicados sobre as espécies de grama estudadas.

Para o primeiro componente principal $\left(\mathrm{Y}_{1}\right)$, os caracteres que mais contribuíram para a discriminação dos tratamentos de trinexapacethyl entre as espécies de gramas foram: porcentagens de epiderme adaxial, bainha do feixe vascular e parênquima, além da espessura foliar. $\mathrm{O}$ componente $\mathrm{Y}_{1}$ apresentou $59,15 \%$ de informação retida, a qual é considerada satisfatória para explicar a contribuição dos caracteres quantitativos analisados, na formação dos agrupamentos dos tratamentos aplicados sobre as quatro espécies de gramas avaliadas. Os caracteres referentes às porcentagens de epiderme abaxial, de feixe vascular, de esclerênquima e o número de feixes apresentaram baixo poder discriminatório.

Quanto ao segundo componente principal $\left(\mathrm{Y}_{2}\right)$, pode-se verificar que a informação retida foi de $24,65 \%$, cujo valor é considerado baixo. Dessa maneira, somente o caráter relacionado com a porcentagem de esclerênquima

Tabela 2 - Coeficientes de correlação entre os oito caracteres anatômicos quantitativos da região da asa de quatro espécies de grama e os dois primeiros componentes principais

\begin{tabular}{|c|l|r|r|}
\hline $\mathrm{N}^{\mathbf{0}}$ & \multicolumn{1}{|c|}{ Caracteres } & \multicolumn{1}{|c|}{$\mathrm{Y}_{1}$} & \multicolumn{1}{c|}{$\mathrm{Y}_{2}$} \\
\hline 1 & \% Epiderme adaxial & 0,9872 & 0,1209 \\
\hline 2 & \% Epiderme abaxial & $-0,0653$ & 0,7672 \\
\hline 3 & \% Bainha do feixe vascular & 0,9949 & 0,0574 \\
\hline 4 & \% Feixe vascular & 0,5754 & $-0,6746$ \\
\hline 5 & \% Esclerênquima & $-0,0916$ & $-0,8240$ \\
\hline 6 & \% Parênquima & $-0,9906$ & 0,0915 \\
\hline 7 & Espessura foliar ( m) & $-0,8734$ & 0,1702 \\
\hline 8 & Número de feixes vasculares & $-0,6387$ & $-0,0700$ \\
\hline & \% Informação retida & 59,15 & 24,65 \\
\hline & \% Informação acumulada & 59,15 & 83,80 \\
\hline
\end{tabular}

apresentou poder de discriminação satisfatório; os demais caracteres avaliados não contribuíram para discriminar os tratamentos aplicados sobre as quatro espécies de grama avaliadas.

Observando a dispersão gráfica referente à análise dos componentes principais (Figura 2), pode-se verificar que os caracteres estruturais quantitativos da região da asa do limbo foliar, que apresentaram maior poder discriminatório, foram os responsáveis pelos agrupamentos formados entre as espécies e os tratamentos com o trinexapac-ethyl, constituindo dessa forma dois grupos principais.

O primeiro grupo foi formado por dois subgrupos, constituídos pelos tratamentos aplicados na espécie $S$. secundatum e pelos tratamentos das doses de trinexapac-ethyl aplicadas em $P$. notatum (subgrupo 1), além dos tratamentos aplicados na espécie A. compressus juntamente com a testemunha de $P$. notatum (subgrupo 2). Portanto, os tratamento aplicados nas espécies de gramas pertencentes ao grupo 1 constituíram subgrupos independentes, uma vez que mostraram alto grau de dissimilaridade entre si. Da mesma maneira, o segundo grupo principal foi constituído pelos tratamentos aplicados na espécie $Z$. japonica.

Conforme verificado na região da quilha do limbo foliar, ambas as doses de trinexapacethyl pertencentes a cada grupo e subgrupos formados para os dados da região da asa apresentaram características mais similares entre si do que em relação às testemunhas, com exceção das doses pertencentes ao grupo 2. Esse resultado indica que a aplicação sequencial de trinexapac-ethyl nas espécies de grama pode alterar alguns caracteres anatômicos da região da asa do limbo foliar.

Comparando os coeficientes de correlação do $\mathrm{Y}_{1}$ na Tabela 2 com a dispersão gráfica da Figura 2, pode-se constatar que as espécies $S$. secundatum, $P$. notatum e A. compressus, com seus tratamentos correspondentes, constituintes do grupo 1, apresentaram os menores valores dos caracteres relativos à epiderme adaxial e à bainha do feixe vascular, bem como os maiores valores dos caracteres relativos ao parênquima e à espessura foliar. O grupo 2 (Z. japonica) apresentou os maiores valores dos 
caracteres relativos à epiderme adaxial e à bainha do feixe vascular, bem como os menores valores dos caracteres referentes ao parênquima e à espessura foliar.

Esses resultados podem explicar a maior similaridade entre as espécies do grupo 1, assim como a alta dissimilaridade que ocorreu na espécie do segundo grupo principal.

As estruturas anatômicas da folha relativas à epiderme, feixe vascular, bainha do feixe vascular, parênquima, esclerênquima, estômatos, tricomas e espessura da folha podem influenciar na deposição, retenção, absorção e translocação de soluções aplicadas sobre as folhas, funcionando como barreiras, e, dessa forma, estar associadas com a maior ou menor resistência ou tolerância de determinada planta aos produtos fitossanitários.

Entre as poucas informações obtidas na literatura, que relacionam os caracteres quantitativos da lâmina foliar com a absorção e translocação de compostos aplicados sobre as folhas, destacam-se os trabalhos realizados por King \& Radosevich (1979), Bukovac \& Petracek (1993), Fagerness \& Penner (1998c), Santos et al. (2002), Ferreira et al. (2002a,b), Procópio et al. (2003) e Tuffi Santos et al. (2006).

Segundo Tuffi Santos et al. (2006), a maior densidade de células epidérmicas pode implicar maior número de regiões mais propensas à penetração de soluções pulverizadas sobre as folhas.

Da mesma maneira, Ervin \& Koski (2001) observaram que a aplicação do trinexapacethyl (270 $\left.\mathrm{g} \mathrm{ha}^{-1}\right)$ sobre a grama Poa pratensis promoveu aumento da densidade das células do mesofilo do limbo foliar, assim como da concentração da clorofila $b$, na ordem de 26,7 e 43,6\%, respectivamente, em relação à testemunha não aplicada e após quatro semanas da aplicação. Contudo, o trinexapac-ethyl não alterou os teores de carboidratos estruturais, como celulose, hemicelulose e lignina. Assim,

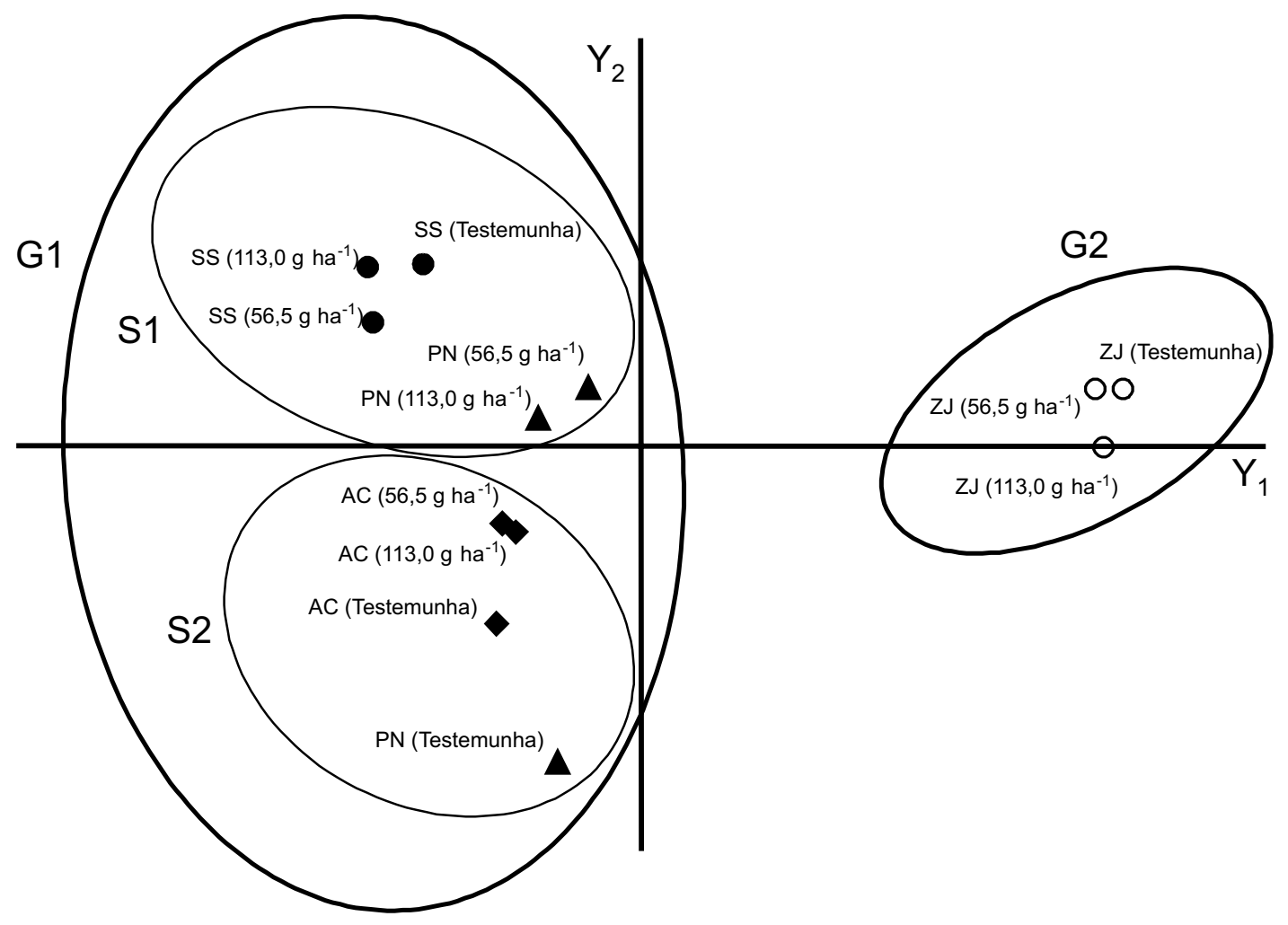

Figura 2 - Dispersão gráfica das quatro espécies de grama e dos tratamentos com trinexapac-ethyl, utilizando-se os dois primeiros componentes principais $\left(\mathrm{Y}_{1}\right.$ e $\left.\mathrm{Y}_{2}\right)$, para o conjunto dos oito caracteres anatômicos quantitativos da região da asa. $\mathrm{AC}=$ Axonopus compressus; $\mathrm{SS}=$ Stenotaphrum secundatum, $\mathrm{PN}=$ Paspalum notatum $;$ e ZJ = Zoysia japonica . G1 = grupo 1; G2 = grupo 2 , $\mathrm{S} 1=$ subgrupo 1 ; e S2 $=$ subgrupo 2. 
esses efeitos promovidos na anatomia das folhas dos gramados podem aumentar a tolerância de várias espécies de grama ao sombreamento e ao estresse hídrico, por apresentarem maior eficiência fotossintética, devido à maior concentração de clorofilas ativas nessas estruturas.

De acordo com Green et al. (1990), a aplicação dos reguladores vegetais flurprimidol (840 $\left.\mathrm{g} \mathrm{ha}^{-1}\right)$ e mefluidide (420 $\mathrm{g} \mathrm{ha}^{-1}$ ) sobre grama $S$. secundatum promoveu redução na evapotranspiração e na superfície foliar em média de 18 e $83 \%$, respectivamente, proporcionando ao gramado maior resistência ao déficit hídrico.

Qian et al. (1998) relataram que aplicações sequenciais de $48 \mathrm{~g} \mathrm{ha}^{-1}$ de trinexapac-ethyl em $Z$. matrella aumentaram a qualidade estética do gramado, favorecendo os processos fisiológicos, como a fotossintese, em cerca de $50 \%$ sob condição de $88 \%$ de sombreamento. Em outro trabalho, Qian \& Engelke (1999) observaram que a aplicação mensal (48 $\left.\mathrm{g} \mathrm{ha}^{-1}\right)$ e bimensal (96 $\mathrm{g} \mathrm{ha}^{-1}$ ) de trinexapac-ethyl aumentou a fotossintese em $Z$. matrella em 48,3 e $41,9 \%$, respectivamente, quando cultivada com $88 \%$ de sombreamento.

Rocha (2001), avaliando espécies de plantas daninhas do gênero Commelina, constatou que $C$. benghalensis, C. vilosa, $C$. diffusa e C. erecta podem ser diferenciadas com base nos caracteres anatômicos quantitativos da folha e do caule, principalmente por aqueles que estão relacionados com a espessura total da folha, o tamanho da nervura mediana e a quantidade de feixes vasculares presentes no caule. Outro fato importante foi que as espécies $C$. benghalensis e $C$. vilosa, mais suscetiveis a herbicidas, apresentaram valores superiores desses caracteres, facilitando, provavelmente, a rápida translocação dos herbicidas que penetram pelos tecidos da folha e do caule, ao passo que C. diffusa e C. erecta, mais tolerantes aos herbicidas, apresentaram menores valores com relação a esses caracteres (Rocha et al., 2007).

Procópio et al. (2003) consideram a cutícula espessa, o alto teor de cera epicuticular, a alta densidade de tricomas, a baixa densidade estomática e a grande espessura da face adaxial da epiderme como as principais barreiras potenciais à penetração de herbicidas em folhas de plantas daninhas. Este trabalho corrobora os dados obtidos por King \& Radosevich (1979), que constataram aumento da absorção de ${ }^{14} \mathrm{C}$-triclopyr em Lithocarpus densiflorusi quando a superficie foliar apresentou baixa quantidade de ceras epicuticulares, alta densidade de estômatos, cutícula delgada e alta densidade de tricomas.

Com base nos resultados obtidos, verificouse que foi possivel agrupar os tratamentos de trinexapac-ethyl utilizados nas diferentes espécies de grama, de forma que aqueles pertencentes a um mesmo agrupamento apresentassem maior similaridade em relação aos demais grupos formados. Concluiu-se que a aplicação sequencial de trinexapac-ethyl alterou algumas estruturas anatômicas da região da quilha (nervura mediana) e da região da asa (compreendida entre a nervura mediana e a margem do limbo foliar) das espécies de grama estudadas.

Contudo, é importante ressaltar a escassez de informações na literatura referentes à utilização de reguladores vegetais nas gramas cultivadas no Brasil, bem como os efeitos das possiveis alterações dos caracteres anatômicos foliares sobre a absorção e translocação de compostos aplicados nas folhas, processos físiológicos como a fotossintese, resistência ao pisoteio, resistência ao estresse hídrico, eficiência de utilização da água de irrigação e diminuição da taxa de evapotranspiração. Portanto, torna-se fundamental a realização de mais estudos que abordem esses assuntos.

\section{LITERATURA CITADA}

ADAMS, R. et al. Studies on the action of the new growth retardant CGA 163935 (cimectacarb). In: KARSEN, C. M.; van LOON, L. C.; VREUGDENHIL, D. (Eds). Progress in plant growth regulation. Dordrecht: Kluwer Academic, 1992. p. $818-827$.

BUKOVAC, M. J.; PETRACEK, P. D. Caracterizing pesticide and surfactant penetration with isolated plant cuticles. Pest. Sci., v. 37, n. 1, p. 179-194, 1993.

BUSH, E. W. et al. Controlling growth of common carpetgrass using selected plant growth regulators. Hortscience, v. 33, n. 4, p. 704-706, 1998.

COSTA, N. V. et al. Características morfológicas de gramas em esposta à aplicação de trinexapac-ethyl. Planta Daninha, v. 27, n. 1 , p. $113-122,2009$. 
DERNOEDEN, P. H. Four-year response of a Kentucky bluegrass-red fescue turf to plant growth retardants. Agron. J., v. 76, n. 5, p. 807-813, 1984.

ERVIN, E. H.; KOSKI, A. J. Trinexapac-ethyl increases Kentucky bluegrass leaf cell density and chlorophyll concentration. Hortscience, v. 36, n. 4, p. 787-789, 2001.

FAGERNESS, M. J.; PENNER, D. Evaluation of V-10029 and trinexapac-ethyl for annual bluegrass seedhead suppression and growth regulation of five cool-season turfgrass species. Crop Sci., v. 12, n. 3, p. 436-440, 1998a.

FAGERNESS, M. J.; PENNER, D. Spray application parameters that influence the growth inhibiting effects of trinexapac-ethyl. Crop Sci., v. 38, p. 1028-1035, 1998b.

FAGERNESS, M. J.; PENNER, D. ${ }^{14}$ C-trinexapac-ethyl absorption and translocation in Kentucky bluegrass.

Crop Sci., v. 38, n. 4, p. 1023-1027, 1998c.

FERREIRA, E. A. et al. Estudos anatômicos de folhas de plantas daninhas. I. Nicandra physaloides, Solanum americanum e Raphanus raphanistrum. Planta Daninha, v. 20, n. 2, p. 159-167, 2002a.

FERREIRA, E. A. et al. Estudos anatômicos de folhas de plantas daninhas. II. Bidens pilosa, Emilia sonchifolia, Agerantum conyzoides e Sonchus asper. Planta Daninha. v. 20, n. 3 , p. $327-335,2002$ b.

FREITAS, F. C. L. et al. Efeitos do trinexapac-ethyl sobre o crescimento e florescimento da grama-batatais.

Planta Daninha, v. 20, n. 3, p. 477-486, 2002.

GERRITS, P. O. The application of glycol metacrylate in histotechnology: same fundamental principles. Germany: Lica Gmgh, 1991. 80 p.

GREEN, R. L. et al. Effects of flurprimidol, mefluidide, and soil moisture on St. Augustinegrass evapotranspiration rate. Hortscience, v. 25, n. 4, p. 469-441, 1990.

HECKMAN, N. L. et al. Heat tolerance of Kentucky bluegrass as affected by trinexapac-ethyl. Hortscience, v. 36, n. 2 , p. $365-367,2001$.

JIANG, H.; FRY, J. Drought responses of perennial ryegrass treated with plant growth regulators. Hotscience, v. 33, n. 2 , p. 270-273, 1998.

JOHANSEN, D. A. Plant microtechnique. New York: McGrow-Hill Book, 1940. 523 p.

JOHNSON, B. J. Frequency of plant growth regulators and mowing treatments: effects on injury and suppression of centipedegrass. Agron. J., v. 85, n. 2, p. 276-280, 1993.
JOHNSON, B. J. Influence of plant growth regulators and mowing two bermudagrasses. Agron. J., v. 86, n. 5, p. 805-810, 1994.

JOHNSON, B. J. Responde of "Tifway" bermudagrass to rate and frequency of flurprimidol and paclobutrazol application.

Hotscience, v. 27, n. 3, p. 230-233, 1992.

JOHNSON, B. J. Growth of "Tifway" bermudagrass following application of nitrogen and iron with trinexapacethyl. Hotscience, v. 32, n. 2, p. 241-242, 1997.

KING, M. G.; RADOSEVICH, S. R. Tanoak (Lithocarpus densiflorus) leaf surface characteristics and absorption of triclopyr. Weed Sci., v. 27, n. 6, p. 599-604, 1979.

LICKFELDT, D. W. et al. Implications of repeated trinexapac-ethyl applications on Kentuchy bluegrass. Agron. J., v. 93, n. 5, p. 1164-1168, 2001

McDANIEL, G. L. et al. Alteration of poinsettia stem anatomy by growth-retarding chemicals. Hortscience, v. 25, n. 4, p. 433-435, 1990.

METCALFE, C. R. Anatomy of the monocotyledons. I. Gramineae. Oxford: Clarendon Press, 1960. 731 p.

MONQUERO, P. A. et al. Controle pelo glyphosate e caracterização geral da superfície foliar de Commelina benghalensis, Ipomoea hederifolia, Richardia brasiliensis e Galinsoga parviflora. Planta Daninha, v. 23, n. 1, p. 123-132, 2005.

O'BRIEN, T. P. et al. Polychmofic staing of plant cellular wall by toluidine blue. Protoplasma, v. 59, n. 2, p. 368-373, 1964.

PROCÓPIO, S. O. et al. Estudos anatômicos de folhas de espécies de plantas daninhas de grande ocorrência no Brasil. III - Galinsoga parviflora, Crotalaria incana, Conyza bonariensis e Ipomoea cairica. Planta Daninha, v. 21, n. 1, p. 1-9, 2003.

QIAN, Y. L. et al. Trinexapac-ethyl restricts shoot growth and improves quality of "Diamond" zoysiagrass under shade. Hortscience, v. 33, n. 6, p. 1019-1022, 1998.

QIAN, Y. L.; ENGELKE, M. C. Influence of trinexapac-ethyl on diamond zoysiagrass in a shade enviroment. Crop Sci. v. 39 , n. 1, p. 202-208, 1999

ROCHA, D. C. Caracterização morfo-anatômica e genética de quatro espécies invasoras de Commelina $\mathrm{L}$. 2001. 110 f. Tese (Doutorado em Ciências Biológicas/ Botânica) - Instituto de Biociência, Universidade Estadual de Paulista, Botucatu, 2001.

ROCHA, D. C. et al. Efeito de herbicidas sobre quatro espécies de trapoeraba. Planta Daninha, v. 25, n. 2, p. 359-364, 2007. 
SANTOS, I. C. et al. Caracteres anatômicos de duas espécies de trapoeraba e a eficiência do glyphosate. Planta Daninha, v. 20 , n. 1, p. $1-8,2002$

TUFFI SANTOS, L. D. et al. Características da epiderme foliar de eucalipto e seu envolvimento com a tolerância ao glyphosate. Planta Daninha, v. 24, n. 3, p. 513-520, 2006.
SNEATH, P. H. A.; SOKAL, R. R. Numerical taxonomy. San Francisco: W. H. Freemam, 1973. 530p.

SOUZA, L. A. et al. Morfologia e anatomia vegetal: técnicas e práticas. Ponta Grossa: UEPG, 2005. 194 p.

TAN, Z. G.; QIAN, Y. L. Light intensity affects gibbrellic acid content in Kentucky bluegrass. Hortscience, v. 38, n. 1, p. 113-116, 2003. 